\title{
Elevated plasma total homocysteine increased the risk of dementia in the elderly
}

Seshadri S, Beiser A, Selhub J, et al. Plasma homocysteine as a risk factor for dementia and Alzheimer's disease. N EnglJ Med 2002 Feb 14;346:476-83.

\section{QUESTION: Is elevated plasma total homocysteine a risk factor for dementia in the elderly?}

Design

Cohort study with a median follow up of 8 years.

\section{Setting}

Framingham, Massachusetts, USA.

\section{Participants}

1092 elderly participants (mean age 76 y, 61\% women) from the Framingham Study cohort who were free from dementia and had plasma total homocysteine concentrations measured at their $20^{\text {th }}$ biennial examination.

\section{Assessment of risk factors}

Baseline data were collected on plasma total homocysteine concentration. Analyses were adjusted for baseline age; sex; apolipoprotein E genotype (93\% of participants); plasma concentrations of folate $(98 \%$ of participants), vitamin $B_{12}$ (85\% of participants), and vitamin $\mathrm{B}_{6}$ (92\% of participants); educational status; history of stroke; cigarette smoking; alcohol intake; diabetes mellitus; systolic blood pressure; and body mass index.

\section{Main outcome measures}

Dementia (DSM-IV criteria, symptoms $>6$ months' duration, and Clinical Dementia Rating scale severity of dementia score $\geq 1$ ) and Alzheimer's disease (NINCDSADRA criteria for definite, probable, or possible Alzheimer's disease). Outcome assessors were blinded to plasma total homocysteine concentrations.

\section{Main results}

Dementia was diagnosed in 111 participants $(10 \%$ of cohort) and, of those, 83 participants ( $8 \%$ of cohort) were diagnosed with Alzheimer's disease. After adjusting for all collected variables using a Cox proportional hazards model $(\mathrm{n}=680)$, risks of dementia (relative risk [RR] 1.4, 95\% CI 1.1 to 1.9) and Alzheimer's disease (RR 1.8, CI 1.3 to 2.5 ) were increased; the RRs were per increment of 1 standard deviation in the log transformed baseline plasma total homocysteine concentration. Hyperhomocysteinemia (plasma homocysteine $>14 \mu \mathrm{mol} / \mathrm{L}$ ) increased the risks of dementia (RR 1.9, CI 1.3 to 2.8) and Alzheimer's disease (RR 1.9, CI 1.2 to $3.0)$.

For correspondence: Dr P A Wolf,

Department of

Neurology, Boston

University School of

Medicine, Boston, $M A$

USA.pawolf@bu.edu

\section{Conclusion}

Elevated plasma total homocysteine was a risk factor for dementia in the elderly.

\section{COMMENTARY}

A risk factor may reveal disease causation; if it suggests a plausible intervention, then it is all the more interesting. Robust risk factors for Alzheimer's disease are now well established: increased age, Down's syndrome, poor education, and Apolipoprotein E status. These risks are not readily amenable to alteration. Biological risk factors that can be altered are especially attractive. Some, such as oestrogen use and middle life vascular risk factors, have accumulating evidence and suggest obvious, if awkward, interventions. The homocysteine story includes a plausible mechanism for causing Alzheimer's disease, and the suggested intervention (dietary folate supplementation) is cheap, easy, and available to all. Folate prevents neural tube defects, which has already led to dietary supplementation in the US.

The study by Seshadri $e t a l$ is hypothesis generating and prospectively studies 1092 participants from the Framingham Study, of whom 111 subsequently developed dementia. The wide confidence intervals of the RRs reported in the study suggest the need for caution in interpreting these results. Can the results be explained because of inadequate adjustment for possible confounding factors? In addition, the findings of Seshadri et al are contrary to those from the other large prospective study of homocysteine and cognition. Some of these uncertainties can be resolved by doing larger studies that provide estimates of the association between homocysteine and dementia in a range of different circumstances (such as at different levels of other risk factors and at different times in the evolution of risk). Analogy with plasma risk factors in cardiovascular disease is instructive. Initial studies overestimated the role of homocysteine, and a recent synthesis of the evidence suggests that it remains uncertain, ${ }^{2}$ even though $>10$ times the amount of information is available for cardiovascular disease than for the dementias. It is important to get the nature of the relation between homocysteine and dementia right because the implications of this link are far reaching.

Bart Sheehan, MRCPsych Seena Fazel, MRCPsych, University Department of Psychiatry Warneford Hospital Oxford, UK

1 Kalmijn S, Launer LJ, Lindemans J, et al. Total homocysteine and cognitive decline in a communityhomocysteine and cognitive decline in a communitybased sample of elderly subject

2 Danesh J, Lewington S. Plasma homocysteine and coronary heart disease: systematic review of published epidemiological studies. J Cardiovasc Risk 1998;5:22932. 